\title{
Asymptotic Performance of Transmit Diversity via OFDM for Multipath Channels
}

\author{
Nadeem Ahmed and Richard G. Baraniuk \\ Department of Electrical and Computer Engineering \\ Rice University, 6100 Main Street \\ Houston, Texas 77005 \\ \{nahmed,richb\}@rice.edu,www.dsp.rice.edu
}

\begin{abstract}
Many wireless systems exploit transmit diversity for more reliable detection of signals at the receiver. To accomplish this, coding is spread across mulitiple transmit antennas. An example of this is the well known "Alamouti transmit diversity", where a very simple coding scherne across multiple transmit antennas allows systems to attain performance similar to systems with multiple receive antennas. The major drawback is that this system only works when a "ffat-fading" model for the channel is assumed; when used in a multipath environment, the system breaks down. Here we show that when the Alamouti code is placed within an OFDM structure, using adjacent frequency bands rather than consecutive symbol intervals, it can asymptotically achieve the same performance in multipath fading as the Alamouti code in flat-fading.

Keywords - transmit diversity, OFDM, frequency selective fading, multipath, multicarrier.
\end{abstract}

\section{INTRODUCTION}

Multipath fading is the fundamental impairment to reliable, high-speed wireless communication [6]. Disruptive channels impair communications by lowering signal-tonoise ratios (SNRs), forcing system designers to compensate by lowering transmission rates and/or tolerating more transmission errors.

Improving the SNR in real-world systems by increasing power or using additional bandwidth is not feasible as these resources are often fixed. Instead, "diversity" (time, frequency, or spatial) is used to improve SNR without violating power or bandwidth constraints. A typical approach is to use multiple antennas at the receiver; the signals from all the branches are weighted and combined in a manner to improve SNR [5].

In some environments, size, cost, and power constraints make it more feasible to add complexity to base stations rather than to mobile units. Hence, a technique for diversity using multiple antennas at the transmitter is highly desirable. A simple, and highly elegant, diversity technique using multiple transmit antennas was first proposed by Alamouti [1].

In maximal ratio receive combining (MRRC), the signals from each of the diversity branches are weighted and combined in a manner that optimizes the SNR [5]. The diversity order of the Alamouti system is equal an MRRC system with two diversity branches [1]. However, the cost of such a system is a $3 \mathrm{~dB}$ loss in SNR, as the diversity is obtained by using multiple transmit rather than receive antennas.

The major drawback of the Alamouti scheme is that it assumes flat-fading in frequency (where the channel is simply a scaled impulse in time) and that it remains constant for consecutive symbol intervals. Practical channels in wireless systems often have frequency-selective multipath components. Here, the channel impulse response is a "waveform" rather than a scaled impulse. The Alamouti scheme cannot deal with such channels, since the assumptions made in [1] break down. As a result the InterSymbol Interference (ISI) introduced by the channel dominates, making errors in transmission much more likely.

A highly desirable system would offer the benefits of the Alamouti technique, but be resistant to the effects of multipath fading. Orthogonal Frequency Division Multiplexing (OFDM) is a multicarrier modulation technique that is often used to combat multipath effects. We can extend the Alamouti scheme by combining it with the OFDM structure, or space-frequency coding, as proposed in [2]. By using adjacent carriers within the OFDM structure, we can obtain the diversity advantage of the Alamouti system, in the presence of multipath fading.

This paper examines this simple space-frequency coding technique and shows that it can asymptotically acheive the same level of performance in frequency selective, multipath channels that the Alamouti technique does in flat-fading.

\section{BACKGROUND}

\section{A. Alamouti Transmit Diversity}

The benefits of diversity has made the use of multiple antennas very attractive. The rationale is that if the antennas are sufficiently separated, then fading affects the signal on each antenna independently, giving the receiver several "looks" at the data, hence improving performance.

The simple transmit diversity first presented by Alamouti works as follows [1]. During each symbol period, two signals are simultaneously transmitted on two sep- 
arate antennas. The signals each see different channels and the sum is received on a single receive antenna. The scheme codes across two antennas and over two consecutive symbol periods. During the first time interval, at time $t$, antenna zero transmits $s_{0}$, and antenna one transmits $s_{1}$. During the next interval, at time $t+T$, antenna zero sends $-s_{1}^{*}$, and antenna one sends $s_{0}^{*}$.

The channel $h_{i}$, for antenna $i$, is assumed to be constant for the two consecutive symbol intervals:

$$
\begin{aligned}
& h_{0}(t) \stackrel{A l m}{=} h_{0}(t+T):=h_{0}=\alpha_{0} e^{j \theta_{0}} \\
& h_{1}(t) \stackrel{A l m}{=} h_{1}(t+T):=h_{1}=\alpha_{1} e^{j \theta_{1}}
\end{aligned}
$$

The received signals, over consecutive symbol intervals, are then

$$
\begin{array}{lll}
r_{0} & \stackrel{A l m .}{=} & r(t)=h_{0} s_{0}+h_{1} s_{1}+h_{0} \\
r_{1} \stackrel{A l m .}{=} & r(t+T)=-h_{0} s_{1}^{*}+h_{1} s_{0}^{*}+n_{1} .
\end{array}
$$

The receiver combines $r_{0}$ and $r_{1}$ in a manner that provides two estimates for the transmitted symbols

$$
\begin{array}{lll}
\widetilde{s}_{0} & \stackrel{A l m .}{=} & h_{0}^{*} r_{0}+h_{1} r_{1}^{*} \\
\widetilde{s}_{1} & \stackrel{A l m}{=} & h_{1}^{*} r_{0}-h_{0} r_{1}^{*}
\end{array}
$$

which when expanded produce estimates

$$
\begin{array}{lll}
\tilde{s}_{0} & \stackrel{A l m .}{=} & \left(\alpha_{0}^{2}+\alpha_{1}^{2}\right) s_{0}+h_{0}^{*} n_{0}+h_{1} n_{1}^{*} \\
\tilde{s}_{1} \stackrel{A l m .}{=} & \left(\alpha_{0}^{2}+\alpha_{1}^{2}\right) s_{1}-h_{0} n_{1}^{*}+h_{1}^{*} n_{0} .
\end{array}
$$

that are then sent to the maximum likelihood detector. An amazing property of this method is that this scheme produces an estimate with a similar form as that of the twobranch MRRC [1].

$$
\begin{array}{ccc}
\widetilde{s}_{0} & \stackrel{M R R C}{=} & \left(\alpha_{0}^{2}+\alpha_{1}^{2}\right) s_{0}+h_{0}^{*} n_{0}+h_{1}^{*} n_{1} \\
\widetilde{s}_{1} & \stackrel{M R R C}{=} & \left(\alpha_{0}^{2}+\alpha_{1}^{2}\right) s_{1}-h_{0}^{*} n_{1}+h_{1}^{*} n_{0} .
\end{array}
$$

\section{B. OFDM Modulation}

OFDM systems divide the transmission bandwidth into a number of smaller subbands, obtained via an orthogonal transformation on a block of data [9]. The receiver performs the inverse transformation to perfectly recover the data, provided there was no channel distortion.

Contemporary OFDM implementations use the Discrete Fourier Transform (DFT) and its inverse IDFT operation for data modulation and demodulation. Figure 1 illustrates a block diagram of a typical OFDM system. In an OFDM system with a DFT of size $N$, there are $N$ individual carriers, or tones, which transmit information. Conceptually, data is buffered and placed on each of these carriers in the frequency domain prior to modulation. The IDFT operation is next performed and a timedomain waveform is sent across the channel. The receiver reverses these operations to recover the data.

For an OFDM system with $N$ subcarriers, the output of the IDFT operation is

$$
x(n)=\frac{1}{\sqrt{N}} \sum_{k=0}^{N-1} X(k) e^{-i \frac{2 \pi n k}{N}}
$$

where $X(k)$ is the data (a QPSK constellation point, for example) on the $k^{\text {th }}$ subcarrier. Each subcarrier is $N$ samples long, i.e., $n \in 0,1, \ldots, N-1$.

The carriers in an OFDM system are orthogonal to one another, in spite of the fact that they overlap in the frequency domain. However, in the presence of multipath interference, the carrier waveforms are distored and hence lose orthogonality with one another. When this occurs, the inverse transformation at the receiver cannot perfectly recover the data.

It is possible to rescue orthogonality by introducing a cyclic prefix (CP) [7]. The CP consists of the final $\nu$ samples of the original $N$ samples to be transmitted, prefixed to the transmitted OFDM block. The length $\nu$ is determined by the length of the channel's impulse reponse. At the receiver, the $\mathrm{CP}$ is discarded and the remaining $N$ samples are the processed by the receiver. The transmission rate of the transceiver is reduced by a factor of $\frac{N}{N+\nu}$,so it is desirable to make $\nu$ as small, or $N$ as large, as possible.

Since we know that the channel is at most length $\nu$, attaching a $\mathrm{CP}$ of length $\nu$ makes the transmitted signal appear periodic to the channel. This allows the system to avoid the interference (both between OFDM blocks and between carriers) for channels that have an impulse reponse shorter than the CP [7]. From the receiver's point of view, the CP transforms the linear convolution of the channel and the overall transmitted block (data and CP) into a circular convolution of the channel and the data portion of the block. Denoting circular convolution by, $\otimes$ the $N$ received data points, $\underline{r}$, the $N$ transmitted constellation points, $\underline{x}$, and the channel impulse response, $\underline{h}$, the overall OFDM system can be expressed as

$$
\begin{aligned}
\underline{r} & =\operatorname{DFT}(\operatorname{IDFT}(\underline{x}) \otimes \underline{h}+\underline{\tilde{n}}) \\
& =\operatorname{DFT}(\operatorname{IDFT}(\underline{x}) \otimes \underline{h})+\underline{n} .
\end{aligned}
$$

The DFT of the white, Gaussian noise vector $\underline{\tilde{n}}$, is $\underline{n}$, also white and Gaussian.

A well-known signal processing result is that the DFT of two cyclically convolved signals is equivalent to the pointwise product of their individual DFTs [4]. This allows the above expression to be rewritten as

$$
\underline{r}=\underline{x} \odot \mathrm{DFT}(\underline{h})+\underline{n},
$$




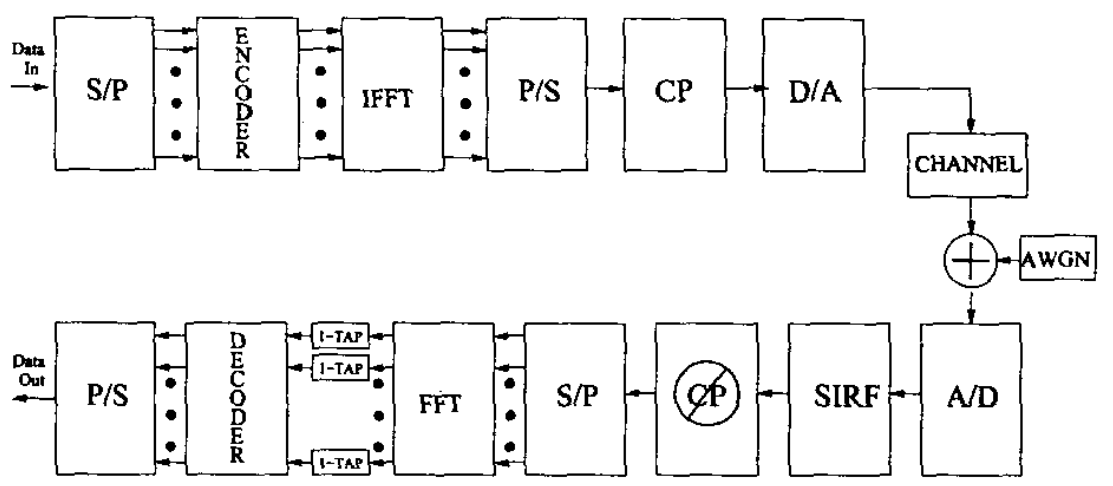

Fig. 1. OFDM system block diagram. Here $\mathrm{S} / \mathrm{P}$ and $\mathrm{P} / \mathrm{S}$ are the serial-to-parallel and paralled-to-serial converters, $\mathrm{A} / \mathrm{D}$ and $\mathrm{D} / \mathrm{A}$ are the analog-todigital and digital-to-analog converters, $\mathrm{CP}$ is the cyclic prefix, and SIRF is the channel equalizer, and AWGN the white gaussian noise.

where $\odot$ represents pointwise multiplication. Conceptually, the received vector is in the frequency domain, as is the transmitted data. The system is equivalent to $N$ para!lel Gaussian channels, which have attenuations given by the vector $D F T(\underline{h})$. Thus, in each subchannel, we have the data that was transmitted multiplied by a complex attenuation factor, the elements of $D F T(\underline{h})$. By using the OFDM structure, we can convert a communication system in the presence of a frequency selective multipath channel into a number of lower bandwidth parallel systems, each with flat-fading.

\section{TRANSMIT DIVERSITY IN MULTIPATH ChanNELS}

To achieve the same diversity order as [1] in frequency selective channels, the simple space-time transmitter diversity technique can be extened to work within the framework of an OFDM system [2]. The OFDM system uses multiple carriers and the CP to mitigate the effect of the multipath channel, at the cost of a slight lower data rate. Effectively the system acts as an $\frac{N}{N+\nu}$ code, as $\nu$ symbols are replicated for the CP for each OFDM block of size $N$.

Rather than assuming the channel is flat in frequency and constant across consecutive intervals, we assume that the channel for each antenna is a waveform across time, which can vary between OFDM blocks. Let us denote the multipath channels as $h_{0}(t)$ and $h_{1}(t)$, and the sampled version as $h_{0}(n)$ and $h_{1}(n)$, respectively. To achieve a diversity advantage over a single antenna system, adjacent frequency bins (or tones) are used in the OFDM system. We assume that the OFDM system has sufficient carriers, such that the channel appears flat across each (see Figure 2). We denote the channels for the tone centered at $f$ for each antenna as

$$
\begin{aligned}
& H_{0}(f)=\alpha_{0} e^{j \theta_{0}}=H_{0} \\
& H_{1}(f)=\alpha_{1} e^{j \theta_{1}}=H_{1}
\end{aligned}
$$

where $H_{0}(f)$ and $H_{1}(f)$ are the DFTs of $h_{0}(n)$ and $h_{1}(n) . \alpha_{i} e^{j \theta_{i}}$ is simply a complex number, the value of the DFT at the tone of interest. We use the notation $H_{i}(f)$, rather than $h_{i}(n)$ for the Alamouti system, since we are modulating data in the frequency domain. The channels on adjacent tones centered at frequency $f+\Delta f$ can be related by

$$
\begin{aligned}
& H_{0}(f+\Delta f)=H_{0}+\Delta H_{0} \\
& H_{1}(f+\Delta f)=H_{1}+\Delta H_{1}
\end{aligned}
$$

we use this notation since the channel attenuation on adjacent tones is not identical. The channels differ at frequency $f+\Delta f$ from those at frequency $f$ by $\Delta H_{0}$ and $\Delta H_{1}$, respectively.

On the tone centered at frequency $f$ antenna zero transmits $s_{0}$ and antenna one transmits $s_{1}$. The adjacent tones, centered at $f+\Delta f$, antenna zero sends $-s_{1}^{*}$, and antenna one sends $s_{0}^{*}$. Since the DFT is of size $N$, within each OFDM block we transmit $\frac{N}{2}$ pairs of symbols. Since each of these pairs are independent of one another, we look at only one for simplicity. The received signal, for the transmitted symbols $s_{0}$ and $s_{1}$, then becomes

$$
\begin{aligned}
r_{0} & =r(f) \\
& =H_{0} s_{0}+H_{1} s_{1}+n_{0} \\
r_{1} & =r(f+\Delta f) \\
& =-\left(H_{0}+\Delta H_{0}\right) s_{1}^{*}+\left(H_{1}+\Delta H_{1}\right) s_{0}^{*}+n_{1}
\end{aligned}
$$

Using the same combiner as in (3) we have the sstimates

$$
\tilde{s}_{0}(N)=\left(\alpha_{0}^{2}+\alpha_{1}^{2}\right) s_{0}+H_{0}^{*} n_{0}+H_{1} n_{i}^{*}
$$




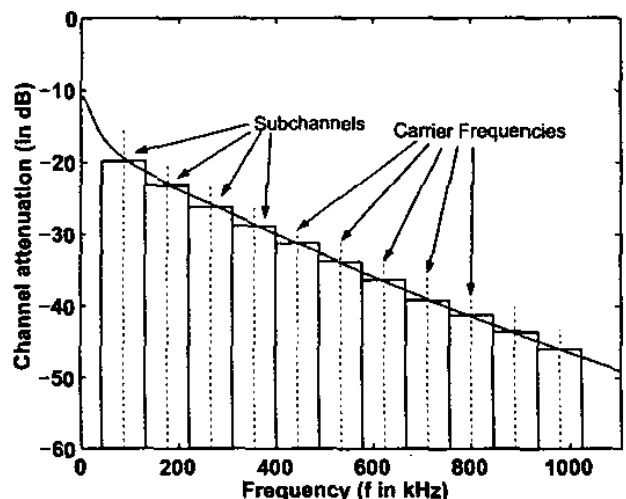

Fig. 2. The size of the DFT chosen in an OFDM system is large enough that the frequency response of the channel is relatively constant across each bin.

$$
\begin{aligned}
& +H_{1}\left(\Delta H_{0}^{*} s_{1}+\Delta H_{1}^{*} s_{0}\right) \\
\widetilde{s}_{1}(N)= & \left(\alpha_{0}^{2}+\alpha_{1}^{2}\right) s_{1}-H_{0} n_{1}^{*}+H_{1}^{*} n_{0} \\
& +H_{0}\left(\Delta H_{0}^{*} s_{1}-\Delta H_{1}^{*} s_{0}\right)
\end{aligned}
$$

We notice that that these estimates are functions of the DFT size $N$, since $\Delta H_{i}$ is a function of $N$. Comparing equations (4) and (12) notice that they are very similar, and as $\Delta H_{i}$ in (12) approach 0 , the expressions become identical.

Fact 1 . If $f(z)$ and $g(z)$ are two complex valued functions of a complex variable $z$, continuous on domain $D$, then $f(z)+g(z)$ is also continuous on $D$ (pp. 57 of [8])

Fact 2. FIR filters have a continuous discrete-time fourier transform (DTFT). This is trivially shown by applying Fact 1 to the expansion of the DTFT of $h(n)$.

Theorem 1. Space-frequency coded OFDM systems achieve the same performance as Alamouti coded spacetime systems as the DFT size $N$ approaches infinity.

Proof:

$$
\begin{aligned}
\lim _{N \rightarrow \infty} \tilde{s}_{0}(N)= & \lim _{\substack{\Delta \rightarrow 0 \\
\tilde{s}_{0}}} \tilde{s}_{0}(N) \\
= & \lim _{\substack{\Delta H_{0} \rightarrow 0 \\
\Delta H_{1} \rightarrow 0}} \tilde{s}_{0}(N) \\
= & \lim _{\substack{\Delta H_{0} \rightarrow 0 \\
\Delta H_{1} \rightarrow 0}}\left(\alpha_{0}^{2}+\alpha_{1}^{2}\right) s_{0}+H_{0}^{*} n_{0}+H_{1} n_{1}^{*} \\
= & \widetilde{s}_{0}+H_{1}\left(\Delta H_{0}^{*} s_{1}+\Delta H_{1}^{*} s_{0}\right)
\end{aligned}
$$

The first equality comes from the fact that as $N$ increases, the interval between samples of the DTFT decreases, hence $\Delta f \rightarrow 0$. The second equality comes from the continuity of the DTFT of $h_{0}(n)$ and $h_{1}(n), H_{0}(f)$ and $H_{1}(f)$ (Fact 2). Since $H_{0}(f)$ and $H_{1}(f)$ are continuous, as $\Delta f \rightarrow 0$ we have $\Delta H_{0} \rightarrow 0$ and $\Delta H_{1} \rightarrow 0$.

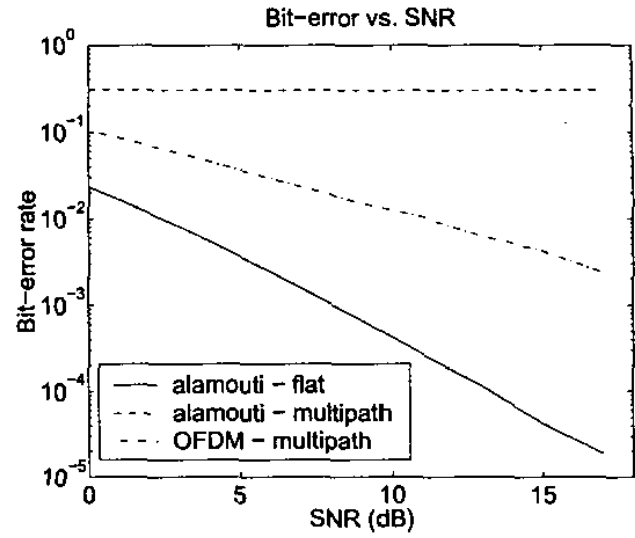

Fig. 3. Bit-error vs. SNR for Alamouti systems in both flat-fading and multipath channels. Performance of single antenna OFDM system in multipath fading included for reference.

\section{Simulation RESUlts}

In all simulations, data is modulated via a 4-point QPSK constellation. In OFDM implementations, each tone carries such a constellation, while in Alamouti systems, each symbol is such a point. In all cases channels are drawn from a Rayleigh distribution and the error rate, averaged over many channel realizations, is plotted against the total transmit power across both antennas.

Figure 3 compares the performance of the Alamouti system in both flat-fading and multipath channels. The first tap of the channel in the multipath case was taken to be the channel estimate for the Alamouti decoder. Comparing the two curves immediately illustrates the effect of multipath. The Alamouti system performs very well in the presence of flat-fading; however, in the presence of multipath the system performs breaks down, regardless of SNR. The performance of the single antenna OFDM system is included as a reference. By comparing it to the Alamouti performance in flat-fading, we can see the effect of diversity. The Alamouti system has an error curve with a greater slope, due to the fact that multiple antennas are used.

Figure 4 illustrates the performance of the spacefrequency coded system. Alamouti performance in flatfading is also included as a reference. We clearly see that as the DFT size of the system increases, the closer the performance of the space-frequency coded system comes to the Alamouti system. We also notice that for the system with a 16-tap channel, the DFT size needs to be larger for the performance to approach the level of the Alamouti system than the system with the 4-tap channel. 


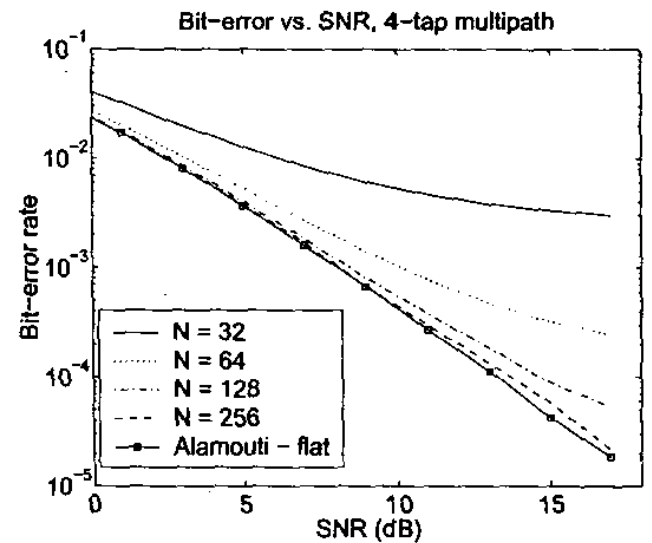

(a)

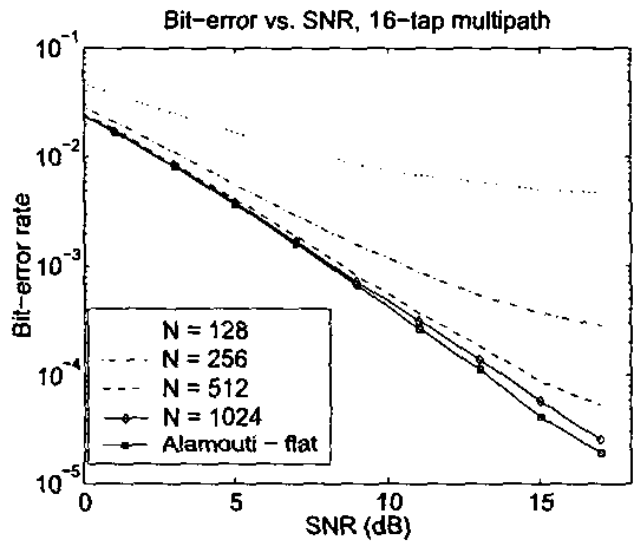

(b)

Fig. 4. Bit-error rate vs. SNR for both 4 (a) and 16 (b) tap multipath channels, plotted for various DFT sizes. As DFT size increases, the performance approaches that of the Alamouti system in flat-fading (also shown).

\section{Discussion AND Conclusions}

The estimates of the transmitted symbols of both the Alamouti system and the space-frequency system in equations (4) and (12) are similar. The space-frequency system has extra interference terms arising from the fact that the channel attenuation on adjacent tones is not identical.

We see in figure 4 that asymptotically the performance of the space-frequency system in multipath channels approaches that of the Alamouti system in flat fading. The interference terms in equation (12) tend to 0 as the DFT size increases. This is precisely what was shown in Theorem 1 and tells us that the performance of spacefrequency coded systems in multipath interference can approach that of Alamouti coded systems in flat-fading. The DFT size can be chosen large enough to bring the space-frequency system arbitrarily close to the Alamouti scheme, a useful property in system design.

Several factors need to be considered in choosing the DFT length. The DFT must be kept large enough that the interference terms in (12) are small, so performance is not adversely affected. The DFT must also be large to keep the rate of the system, $\frac{N}{N+\nu}$, as large as possible. However larger DFT sizes introduce longer latency for the system, since OFDM blocks of size $N$ are processed together. In applications where latency is critical, this needs to be considered. Therefore we have an engineering tradeoff. The DFT size needs to be carefully chosen for the application at hand; large enough for good error performance and a small rate loss, while small enough to minimize latency.

The great advantage of the Alamouti scheme is that is provides a simple technique for gaining a diversity advantage over single antenna systems, while keeping the added complexity of the system at the transmitter rather than the receiver. However, in the presence of multipath interference, the system breaks down. Using the Alamouti code within the OFDM framework addresses this issue. The main contribution of this paper shows that asymptotically the performance of space-frequency coded systems in multipath channels can reach that of Alamouti coded system in flat-fading. This is of great benefit, as we manage to keep the diversity advantage of Alamouti's system without the overly restrictive flat-fading model.

\section{REFERENCES}

[1] S. Alamouti, "A Simple Transmit Diversity Technique for Wireless Communications", IEEE Journal on Select Areas in Communications, Vol. 16, No. 8, pp 1451-1458, October 1998.

[2] K.F. Lee, D.B. Williams “ A space-frequency transmitter diversity technique for OFDM systems" in Global Telecommunications Conference, 2000. GLOBECOM '00 pp. 1473-1477 vol.3, 2000.

[3] Nadeem Ahmed, "Joint Detection Strategies for Orthogonal Frequency Division Multiplexing," M.S. Thesis, ECE Department, Rice University, April 2000.

[4] J.G. Proakis and D. Manolakis, Digital Signal Processing. Principles, Algorithms, and Applications. New Jersey: Prentice Hall, 1996.

[5] T.S. Rappaport, Wireless Communications. Principles and Practices. New Jersey: Prentice Hall, 1996.

[6] W.C. Jakes, Microwave Mobile Communications. New Jersey: Wiley, 1974

[7] A. Peled and A. Ruiz, "Frequency domain data transmission using reduced complexity algorithms", in IEEE Int. Conf. Acoust., Speech. Signal Processing. - ICASSP '80, (Denver, CO), pp. $964-967,1980$.

[8] J.H. Mathews and R.W. Howell, Complex Analysis for Mathematics and Engineering. Iowa: Wm. C. Brown Publishers, 1996.

[9] J.M. Cioffi A Mulficarrier Primer. Amati Communications Corporation and Stanford University. 